\title{
Plastic bronchitis in a toddler born preterm
}

\author{
jinfang yuan ${ }^{1}$, ling $\mathrm{Liu}^{1}$, wei Zhou ${ }^{1}$, xiaomei Tong ${ }^{1}$, and tongyan Han $^{1}$ \\ ${ }^{1}$ Peking University Third Hospital
}

September 11, 2020

\begin{abstract}
Plastic bronchitis is identified by the presence of branching mucoid bronchial casts that obstruct airways. It is an uncommon condition, which was more described in lung disease and post-surgery congenital heart disease. We reported a case of a 26month-old boy born at $29+6$ weeks who presented with cough, wheezing and respiratory distress secondary to atelectasis of the left lung. After admission, the patient was treated with intravenous injection of azithromycin and methylprednisolone, inhaled bronchodilators, inhaled budesonide with short-acting beta-agonists and inhaled nebulized N-acetylcysteine. Dramatically, the patient expelled large casts, shaping as the tracheobronchial tree after a severe cough just on the night before the bronchoscopy. In toddlers who were born preterm with recurrent wheezing, the discharge of airway mucus secretion is altered, and the possibility of bronchial plastics needs to be alert.

Plastic bronchitis in a toddler born preterm

Jinfang Yuan M.D, Ling Liu M.D, Wei Zhou M.D, Xiaomei Tong M.D, Tongyan Han M.D

Department of Pediatric, Peking University Third Hospital, Beijing, China
\end{abstract}

\section{Correspondence}

Tongyan Han, M.D, Department of Pediatrics, Peking University Third Hospital, Beijing 100191, China

Email:tongyanhan@qq.com

\section{Key words}

Plastic Bronchitis, Preterm Infant, Wheezing

Plastic bronchitis in a toddler born preterm

AbstractPlastic bronchitis is identified by the presence of branching mucoid bronchial casts that obstruct airways. It is an uncommon condition, which was more described in lung disease and post-surgery congenital heart disease. We reported a case of a 26 -month-old boy born at $29^{+6}$ weeks who presented with cough, wheezing and respiratory distress secondary to atelectasis of the left lung. After admission, the patient was treated with intravenous injection of azithromycin and methylprednisolone, inhaled bronchodilators, inhaled budesonide with short-acting beta-agonists and inhaled nebulized N-acetylcysteine. Dramatically, the patient expelled large casts, shaping as the tracheobronchial tree after a severe cough just on the night before the bronchoscopy. In toddlers who were born preterm with recurrent wheezing, the discharge of airway mucus secretion is altered, and the possibility of bronchial plastics needs to be alert.

\section{Introduction}

More than 1 in 10 of the world's infants, of more than 15 million children, was born prematurely ${ }^{1}$. As the second leading cause of death in children younger than 5 years of age, prematurity remains a global health problem $^{1}$. The most immature preterm infants are more likely to need ventilation support and supplemental 
oxygen because of their immature lung. Prematurity followed with amnion infection, growth restriction, oxygen toxicity, and volutrauma and barotrama from mechanical ventilation may interrupt normal pulmonary alveolarization and vascularization development and thus create a clinical scenario of lung injury with pathophysiological effects that can extend beyond infancy into adulthood ${ }^{2,3}$. Instead of the eosinophil-mediated inflammation and atopy typical of asthma, the survivors of preterm birth of recurrent broncho-obstructive symptoms result from abnormal growth and development of the architecture of the lung ${ }^{4}$. We report a case of lung atelectasis due to plastic bronchitis in a 26 -month-old toddler who was born at $29^{+6}$ weeks gestational age.

\section{Case presentation}

A 26-month-old boy was admitted to our hospital in September, 2019, with complaint of coughing for two days, fever and wheezing for one day.

\section{Birth History}

The patient was born at same hospital at 29 weeks 6 days' gestation to a 31-year-old primigravid mother by cesarean section. His mother had got preterm premature rupture of membranes (pPROM) for 22 days, and the placental pathology after birth suggested mild stage I chorioamnionitis (CAM). His birth weight was 1230g (50th percentile), and the 1-minute and 5-minute Apgar scores were 8 and 9, respectively. He had spontaneous breathing after birth and was supported by nasal continuous positive airway pressure (nCPAP) and transferred from the operating room to the neonatal intensive care unit (NICU) in a transfer shuttle. He was diagnosed as transient tachypnea syndrome received nCPAP for 24 days with fraction of inspired oxygen $\left(\mathrm{FiO}_{2}\right)<0.35$. During the first 72 hours after birth, the nCPAP level was kept within at $6 \mathrm{cmH}_{2} \mathrm{O}$, then it was titrated to 4 to $5 \mathrm{cmH}_{2} \mathrm{O}$ to achieve the lowest $\mathrm{FiO}_{2}$ level. The boy was treated with cefoperazone sodium and sulbactam sodium for 10 days due to the suspected amnion infection. He was on nasogastric feeding with own mother's milk and premature infant formula, supplemented partial parenteral nutrition in the first 25 days during hospitalization. At discharge, he was on 36 weeks corrected gestational age with body weight $2260 \mathrm{~g}$ (10th percentile).

Follow-up

The boy was followed in the healthcare center in our department. He had moderate speed of catch-up growth (Figure 1). He had eczema since 5 months after birth. And upper respiratory tract infection 3 times during infancy. His body weight was $10.1 \mathrm{~kg}$ and body length was $73.5 \mathrm{~cm}$ at corrected 8 months. The breast feeding was stopped at corrected 15 months because the mother was back to work. Only about $200 \mathrm{ml}$ formula milk was supplement to the diet except for three meals per day. His body weight was $12.8 \mathrm{~kg}$ and body length was $89 \mathrm{~cm}$ at corrected 17 months. Then, he had suffered from repeated wheezing since 18-month-old and diagnosed as bronchial asthma in other hospital. Since then, he had received intermittent inhalation therapy with budesonide and terbutaline. Four weeks before admission, he had an episode of wheezing and thoracic radiograph showed bronchitis changes without other abnormal findings.

After admission, full clinical examination including general, cardiac, chest and abdominal was performed. On examination, the patient appeared lethargy and slightly uncomfortable. The temperature was 38.6, the heart rate was 155 beats per minute, the respiratory rate was 50 breaths per minute, and the transcutaneous oxygen saturation $\left(\mathrm{SpO}_{2}\right)$ was $92 \%$ while he was breathing ambient air. Signs of respiratory distress were observed and presence of inspiratory retractions. Chest auscultation was done by the same physician during the hospital stay. Abnormal auscultatory findings included diminished breath sounds and presence of bronchial breath sounds fine crepitations in the left lung, and presence of wheezing and crackles in the right lung. The remainder of the examination was normal.

Results of laboratory tests are shown in Table 1. The white blood cells count was normal with slightly increased neutrophils proportion. Liver function and kidney function were normal. Arterial blood gas analysis showed hypoxemia. Antibody quantification of mycoplasma pneumoniae was negative.

After the patient was admitted, intravenous injection azithromycin $(10 \mathrm{mg} / \mathrm{kg})$ and methylprednisolone were 
administered. Supplemental oxygen was given. The oxygen saturation ranged from 92 to 95 percent while the patient was breathing 100 percent oxygen. Tachypnea persisted, with minimal retractions and paroxysmal acute cough. A chest radiograph revealed air trapping with hyperinflation in the right lung and opacification of the left upper lobe which could represent atelectasis (Figure 2). He received inhaled budesonide 1mg ipratropium bromide $250 \mu \mathrm{g}$, and terbutaline $2.5 \mathrm{mg}$ four times a day.

On the second day, a computed tomographic (CT) scan of the chest confirmed superior lobe of left lung atelectasis, and the bronchial openings of the upper and lower lobes disappeared (Figure 3). We continued his treatment with inhaled budesonide $1 \mathrm{mg}$ ipratropium bromide $250 \mu \mathrm{g}$, and terbutaline $2.5 \mathrm{mg}$ four times a day and started nebulized Nacetylcysteine $(2.5 \mathrm{~mL} 20 \%$ solution $)$ twice a day. The bronchoscopy was planned on the morning of next day.

During the second night in the hospital, after a severe cough, the patient expelled large casts, sticky whitish secretions, shaping as the tracheobronchial tree (Figure 4). After the cough, inspiratory retractions were disappeared and the respiratory rate slowed down to 30 breaths per minute. Coarse breath sounds were heard in both lungs with fine crepitations and wheezing.

On the third day, chest-X ray reexamined normal permeability in both lungs. Then the bronchoscopy was canceled. Chest auscultation was clear on the fifth day. The patient was discharge home. He is always followed-up in the pediatric pulmonary clinic.

\section{Discussion}

This study represents a report of case that substantiates the airway casts produced by a toddler with preterm birth history and asthma. On physical exam, wheezing, decreased breath sounds and respiratory distress were observed in this patient. After treatment with inhaled budesonide, ipratropium bromide, terbutaline and nebulized $\mathrm{N}$-acetylcysteine, the patient spontaneously expectorated casts without bronchoscopy.

Plastic bronchitis is an uncommon condition, characterized by the formation of tracheobronchial airway casts, which are partially or fully block the bronchial lumen. It is mainly associated with underlying congenital heart disease or lung diseases ${ }^{5}$. As to lung diseases, it has been associated with asthma, allergic bronchopulmonary aspergillosis, mycoplasma pneumoniae,influenza B virus infection and pulmonary tuberculosis, etc ${ }^{6-8}$. Clinically, patient with plastic bronchitis presents with dyspnea, wheezing, or pleuritic chest pain, and may have fever. Chest x-ray and CT findings are often non-specific including opacity or infiltrate 9,10 .

The mechanism of casts' formation remains unclear for the inflammatory casts in lung disease. In patients with asthma, previous studies hypothesis that the cause of casts is likely related to chronic inflammation and its attendant neutrophilic and eosinophilic airway infiltration, with decreased mucociliary clearance, the airways become occluded with eosinophils and neutrophils in a mucinous background ${ }^{9,11}$. There have been several case reports of plastic bronchitis caused by mycoplasma pneumoniae ${ }^{8,12}$. In this case, four weeks before this acute episode, the chest X-ray was normal, indicating the acute infection was the precipitating factor. The disease onset was in the autumn with low to moderate grade of fever. The blood routine examination was normal, and the application of macrolide anti-infection was effective. Thus, we consider the high possibility of mycoplasma pneumoniae infection in this case, in spite of the antibody was negative which may result from the earlier sample time on the third day of disease onset.

During the 24 to 38 weeks of gestation age, which is the saccular phase of lung development, the relationships between the air spaces, capillaries, and mesenchyme takes on more significance ${ }^{13}$. The alveolocapillary membrane is sufficient to participate in gas exchange until approximately 24 weeks of gestation age. For the very low birth weight infants (with birth weight less than 1500g) as this case or the other, are at higher risk because they have very few vessels and alveoli developed at birth. The pulmonary inflammatory response may have been initiated in utero, in the setting of chorioamnionitis. Chorioamnionitis is acute inflammation of the membranes and chorion of the placenta, commonly due to ascending polymicrobial bacterial infection, which leads to preterm premature rupture of membranes (pPROM). The earlier and more serious the exposure to 
chorioamnionitis is, the more immature and disrupted the lung structure would be ${ }^{14,15}$. The initiation of inflammation appears to cause impairment of the growth of alveoli and of the microvasculature. The boy's mother had got pPROM and chorioamnionitis, which may disrupt the offspring's lung alveolarization and vascularization during infancy and childhood.

Wheezing was related to degree of premature birth, increased gestation should improve the infant's respiratory health up to 2 years of age ${ }^{16}$. Recurrent wheeze in infants and toddlers is associated with small airway calibre, low lung function and airway inflammation ${ }^{17}$. In this case, the potential impaired pulmonary function and the acute attack triggering by infection may play important roles in the plastic bronchitis.

On the treatment ${ }^{18}$,flexible or rigid bronchoscopy is most often used for cast removal. Other medication options include aerosolized fibrinolytics, such as urokinase, and inhaled mucolytics, such as acetylcysteine and dornase alfa. Mucolytics appear to be more useful in inflammatory casts, as the mucus contains. In those with lung diseases involving bronchial hyper-reactivity, treatment is based on the use of inhaled and systemic corticosteroids. In this case,the spontaneous expulsion of casts could prove effectively more flexible plugs, and the inhaled N-acetylcysteine also played a role ${ }^{19}$.

In conclusion, we report an exceptional case of a toddler born prematurely provoked plastic bronchitis. In the recurrent attacks of wheezing toddlers, the acute respiratory tract infection disturbs the airway barrier and may induce bronchial plastics, especially in those born prematurely.

\section{Reference}

1. Blencowe H, Cousens S, Oestergaard MZ, Chou D, Moller AB, Narwal R, Adler A, Garcia CV, Rohde S, Say L, Lawn JE, et al. National, regional, and worldwide estimates of preterm birth rates in the year 2010 with time trends since 1990 for selected countries: a systematic analysis and implications. Lancet. 2012;379:2162-2172.

2. Schittny JC. Development of the lung. Cell Tissue Res. 2017; 367:427-444.

3. Urs R, Kotecha S, Hall GL, Simpson SJ. Persistent and progressive long-term lung disease in survivors of preterm birth. Paediatr Respir Rev. 2018;28:87-94.

4. Been JV, Lugtenberg MJ, Smets E, van Schayck CP, Kramer BW, Mommers M, Sheikh A. Preterm Birth and Childhood Wheezing Disorders: A Systematic Review and Meta-Analysis. PLoS Med. 2014;11(1): e1001596.

5. M Seear, H Hui, F Magee, D Bohn, E Cutz.Bronchial casts in children: a proposed classification based on nine cases and a review of the literature. Am J Respir Crit Care Med. 1997;155(1):364-70.

6. Kirito Y, Matsubayashi T, Ohsugi K. Plastic bronchitis: Three cases caused by influenza B virus Yamagata lineage. Pediatr Int. 2019;61(4):421-423.

7. Panchabhai TS, Mukhopadhyay S, Sehgal S, Bandyopadhyay D, Erzurum SC, Mehta AC. Plugs of the Air Passages: A Clinicopathologic Review Chest. 2016;150(5):1141-1157.

8. Cai XF, Sun JM, Li WB, Cheng HB. Clinical analysis of severe plastic bronchitis in 8 children. Chin Crit Care Med. 2016;28(1):73-75.

9. Kunder R, Kunder C, Sun HY, Berry G, Messner A, Frankovich J, Roth S, Mark J. Pediatric plastic bronchitis: case report and retrospective comparative analysis of epidemiology and pathology. Case Rep Pulmonol. 2013;2013:649365.

10. Aram A, Dalal B. The diagnosis and management of plastic bronchitis: a systematic literature review. Chest. 2019;156(4):A1022.

11. Madsen P, Shah SA, Rubin BK. Plastic bronchitis: new insights and a classification scheme. Paediatr Respir Rev. 2005;6(4):292-300.

12. Huang LL, Li RH, Li J, Chen HJ, Peng SM. Association of YKL-40 in bronchoalveolar lavage fluid with airway damage in children with Mycoplasma pneumoniae pneumonia. Chin J Contemp Pediatr. 2019; 21(12): 1188-1192.

13. Smith LJ, McKay KO, Asperen PP, Selvadurai H, Fitzgerald DA. Normal Development of the Lung and Premature Birth. Paediatr Respir Rev. 2010; 11(3):135-142.

14. Morita M, Tanaka K, Matsumura S, Tamura M, Namba F. Perinatal Factors Associated With Bub- 
bly/Cystic Appearance in Bronchopulmonary Dysplasia: A Nationwide, Population-Based Cohort Study in Japan. J Matern Fetal Neonatal Med. 2019 ; 18:1-6.

15. Eduardo VM, María AF, Amro MT, Degraeuwe P, Zimmermann LJ, Kramer BW, Villamor E. Association of Chorioamnionitis With Bronchopulmonary Dysplasia Among Preterm Infants: A Systematic Review, Meta-analysis, and Metaregression. JAMA Netw Open. 2019;2(11):e1914611.

16. Ünal S, Kaya A, Bilgin L, Misirlioğlu E, Kocabaş CN. Wheezing, asthma, and atopy in premature infants at 2 years of age. Turk J Med Sci. 2017;47(2):607-613.

17. Christopher WC, Sailesh K, Sarah JK. Fractional exhaled nitric oxide in preterm-born subjects: A systematic review and meta-analysis. Pediatr Pulmonol. 2019;54(5):595-601.

18. Pérez Ruiz E, López Castillo MC, Caro Aguilera P, Pérez Frías J. Management and Treatment of Pediatric Plastic Bronchitis. Arch Bronconeumol. 2017;53(8):467-468.

19. Kumar A, Jat KR, Srinivas M, Lodha R. Nebulized N-Acetylcysteine for Management of Plastic Bronchitis. Indian Pediatr. 2018;55(8):701-703.

\section{Hosted file}

Table1. Laboratory tests.docx available at https://authorea.com/users/356856/articles/479636plastic-bronchitis-in-a-toddler-born-preterm

\section{Weight-for-age BOYS}

8irth to 2 years (percentiles)

\section{(k) Worid Menth}

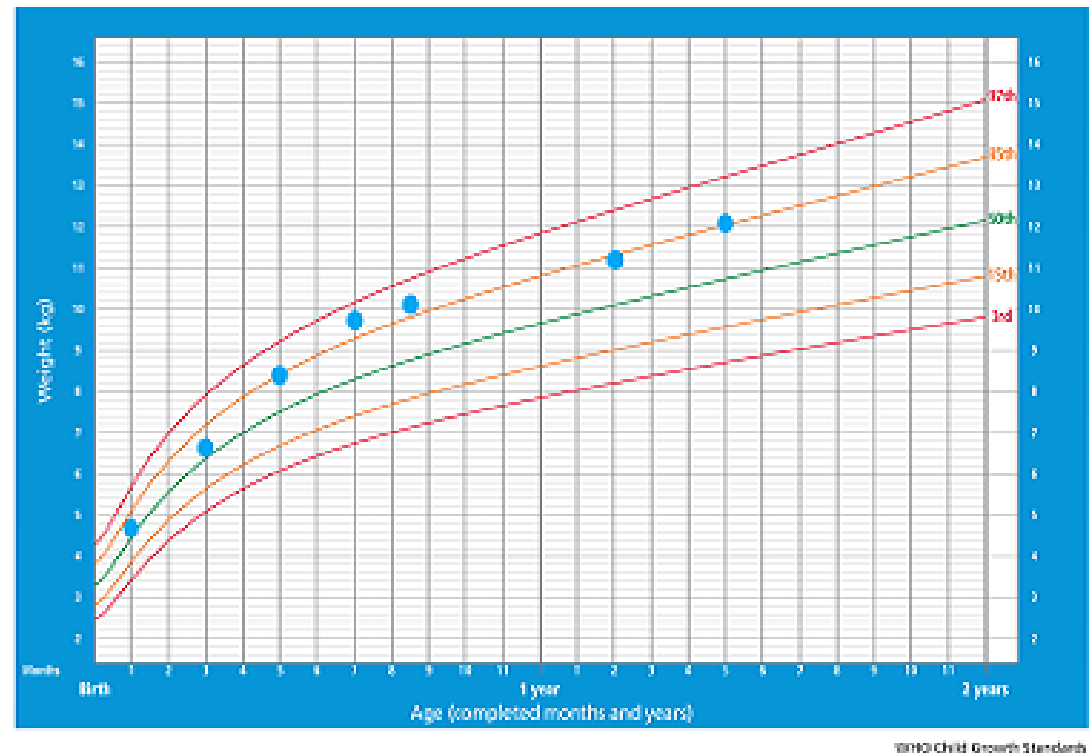



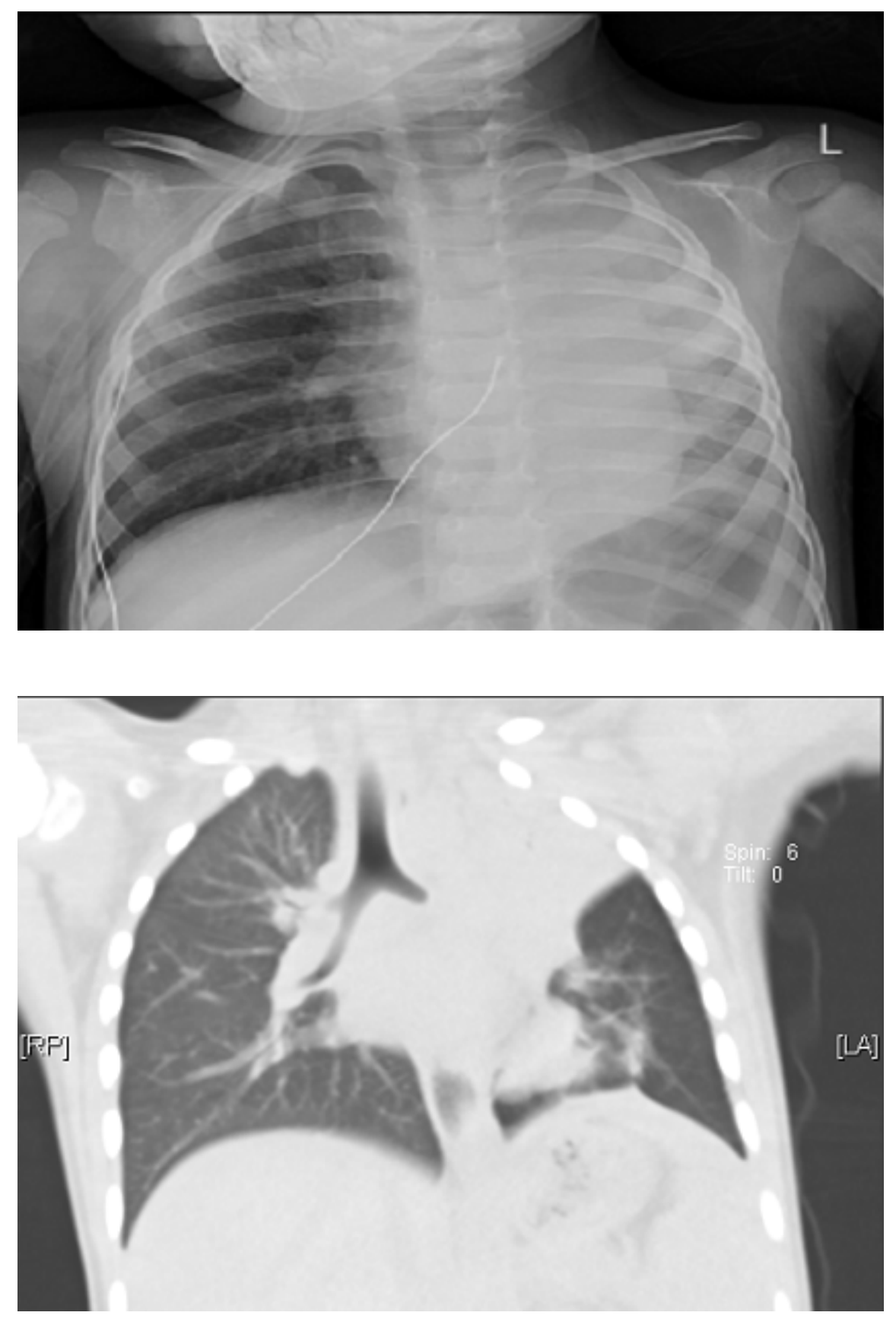


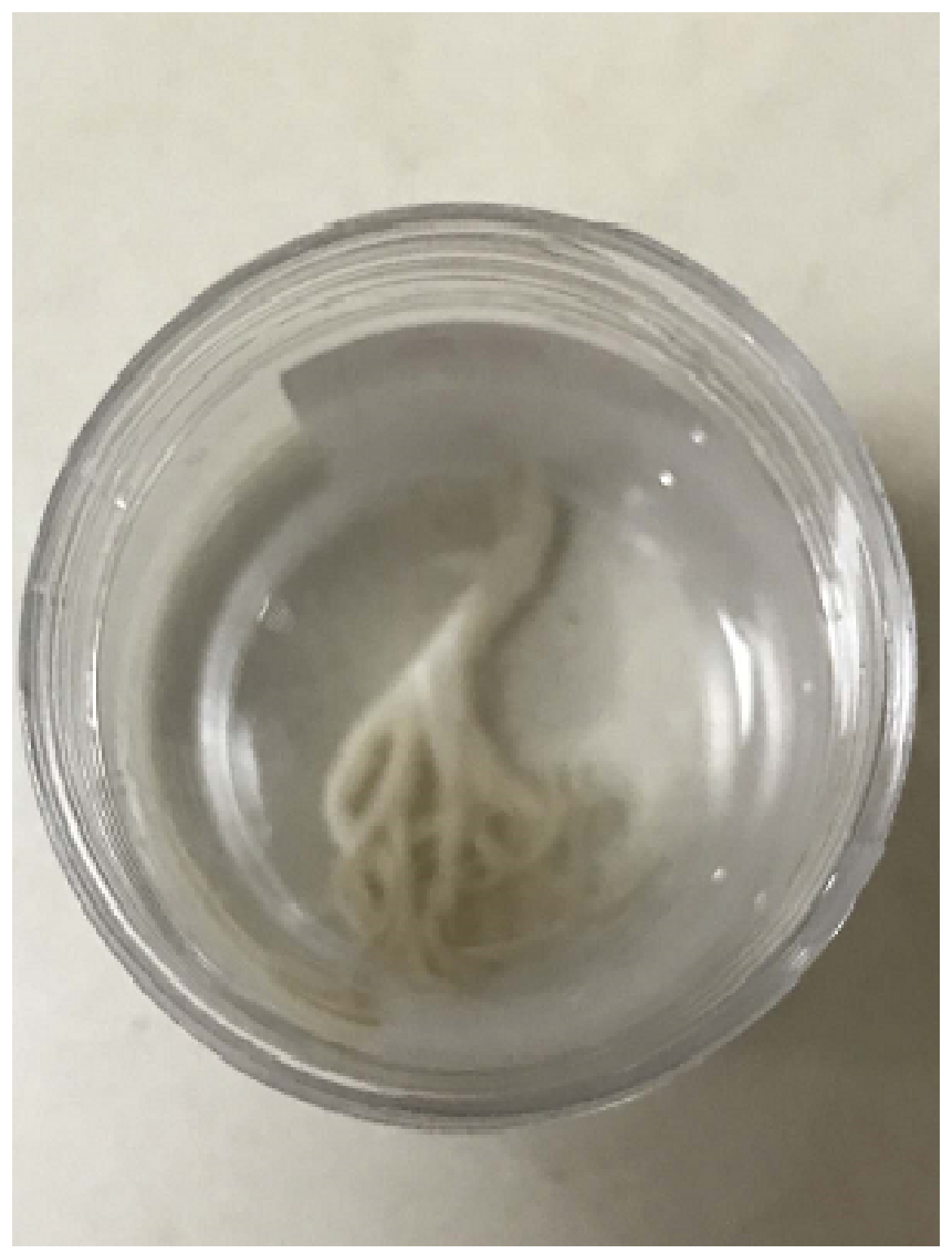

